\title{
Frequency-Domain Gray-Box Identification of Industrial Robots
}

Erik Wernholt, Stig Moberg

Division of Automatic Control

E-mail: erikw@isy.liu.se, stig@isy.liu.se

29th October 2007

Report no.: LiTH-ISY-R-2826

Submitted to 17th IFAC World Congress, Seoul, Korea

Address:

Department of Electrical Engineering

Linköpings universitet

SE-581 83 Linköping, Sweden

WWW: http://wwW. control.isy.liu.se

AUTOMATIC CONTROL

REGLERTEKNIK

LINKÖPINGS UNIVERSITET

Technical reports from the Automatic Control group in Linköping are available from http://www. control.isy.liu.se/publications. 


\begin{abstract}
This paper considers identification of unknown parameters in elastic dynamic models of industrial robots. Identifying such models is a challenging task since an industrial robot is a multivariable, nonlinear, resonant, and unstable system. Unknown parameters (mainly spring-damper pairs) in a physically parameterized nonlinear dynamic model are identified in the frequency domain, using estimates of the nonparametric frequency response function (FRF) in different robot configurations/positions. The nonlinear parametric robot model is linearized in the same positions and the optimal parameters are obtained by minimizing the discrepancy between the nonparametric FRFs and the parametric FRFs (the FRFs of the linearized parametric robot model). In order to accurately estimate the nonparametric FRFs, the experiments must be carefully designed. The selection of optimal robot configurations for the experiments is also part of the design. Different parameter estimators are compared and experimental results show the usefulness of the proposed identification procedure. The weighted logarithmic least squares estimator achieves the best result and the identified model gives a good global description of the dynamics in the frequency range of interest.
\end{abstract}

Keywords: System identification, multivariable systems, nonlinear systems, closed-loop identification, frequency response methods, industrial robots 


\section{Introduction}

Accurate dynamic models of industrial robots are needed for mechanical design, performance simulation, control, supervision, diagnosis, and so on. The industrial robot poses a challenging modeling problem both due to the system complexity and the required model accuracy. Usually a robot has six joints (also called axes), see Figure 1, with coupled dynamics, giving a truly multivariable system. The dynamics is nonlinear, both with respect to the rigid body dynamics and other things such as non-ideal motors and sensors, and a transmission with friction, backlash, hysteresis, and nonlinear stiffness. The system is resonant due to elastic effects and, in addition, experimental data must usually be collected while the robot controller is operating in closed loop since the system is unstable.

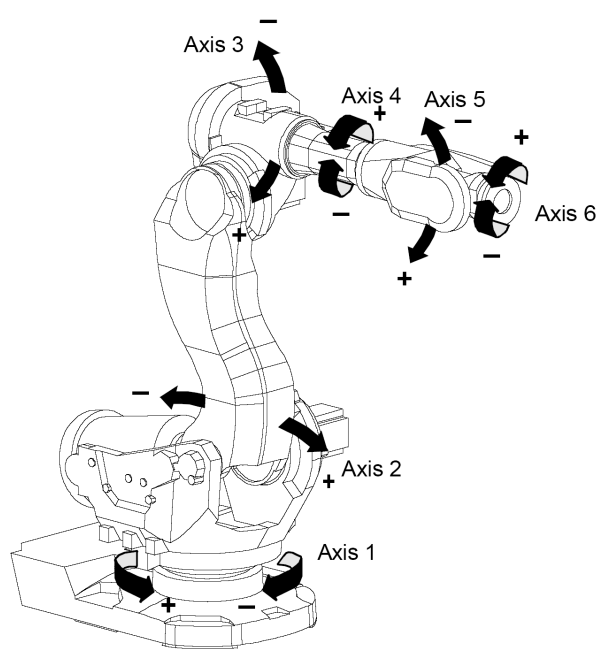

Figure 1: The ABB manipulator IRB6600.

Historically, the dynamic models used for control are either entirely rigid (An et al., 1988), or only flexible joint models are considered, i.e., elastic gear transmission and rigid links (Albu-Schäffer and Hirzinger, 2000; Spong, 1987). The trend in industrial robots is toward lightweight robot structures with a reduced mass but with preserved payload capabilities. This is motivated by cost reduction as well as safety issues, but results in lower mechanical resonance frequencies inside the controller bandwidth. The sources of elasticity in such a manipulator are, e.g., gearboxes, bearings, elastic foundations, elastic payloads, as well as bending and torsion of the links. In Öhr et al. (2006) it is shown that there are cases when these other sources of flexibilities can be of the same order as the gearbox flexibilities for a modern industrial robot. Accurate dynamic models that also describe these elastic effects are therefore needed in order to obtain high performance. These models are, however, very difficult to use for robot control, where, e.g., feedforward control involves solving a DAE, but could in the future improve the performance (Moberg and Hanssen, 2007). 


\section{Problem Description}

The main problem considered in this paper is about identification of unknown parameters in a nonlinear dynamic model of an industrial robot. The model must be global, i.e., valid throughout the whole workspace (all robot configurations/positions), as well as elastic, which here means that resonances due to elastic effects are captured by the model. The elastic effects are modeled through a lumped parameter approach (Khalil and Gautier, 2000) where each rigid body is connected by spring-damper pairs (see also Section 3 ). The model is of gray-box type (a physically parameterized model) and the rigid body parameters of the model are usually assumed to be known from a CAD model or prior rigid body identification. The main objective is identification of elasticity parameters (spring-damper pairs) but other parameters can be added, such as the location in the robot structure of the spring-damper pairs and a few unknown rigid body parameters. It is also possible to include nonlinear descriptions of selected quantities (e.g., the gearbox stiffness) and identify those by a linearization for each position (the terms robot configuration and position are used interchangeably in this paper).

The real challenge for system identification methods is that the industrial robot is multivariable, nonlinear, unstable, and resonant at the same time. Usually, in the literature, at least one of the first three topics is left out. Identification of such a complex system is therefore a huge task, both in finding suitable model structures and efficient identification methods.

One solution could be to apply a nonlinear prediction error method (Ljung, 1999, pp. 146-147), where measured input-output data are fed to the model and the predicted output from the model is compared with the measured output. This has been treated in Wernholt and Gunnarsson (2006a) for axis one of the industrial robot, which means a stable scalar system (axis one is not affected by gravity). Extending these results to a multivariable and unstable system would involve, for example: finding a stable predictor, numerical problems, and handling large data sets. The last two problems stem from the fact that the system is resonant and numerically stiff, as well as large in dimension both with respect to the number of states and parameters. In addition comes also the choice of model structure (parameters) and handling local minima in the optimization. Apart from all these problems, such a solution would really tackle our main problem.

Due to the complexity of the industrial robot, it is common practice to estimate approximate models for various purposes. By, for example, using a lowfrequency excitation, elastic effects have a minor influence and a nonlinear model of the rigid body dynamics can be estimated using least squares techniques. This is a much studied problem in the literature, see, e.g., Kozlowski (1998) for an overview. Taking elastic effects into account makes the identification problem much harder. The main reason is that only a subset of the state variables now are measured such that linear regression cannot be used. One option could be to add sensors during the data collection to measure all states, even though accurate measurements of all states are not at all easy to obtain (if even possible) and such sensors are probably very expensive (for example laser trackers).

It is common to study the local dynamic behavior around certain operating points (also called positions in the paper) and there estimate parametric or nonparametric linear models (see, e.g., Albu-Schäffer and Hirzinger, 2001; Behi 
and Tesar, 1991; Johansson et al., 2000; Öhr et al., 2006). One application area for these linear models is control design, where a global (feedback) controller is achieved through gain scheduling. The linear models can also be used for the tuning of elastic parameters in a global nonlinear robot model, which is the adopted solution in this paper:

- The local behavior is considered by estimating the nonparametric frequency response function (FRF) of the system in a number of positions.

- Next, the nonlinear parametric robot model is linearized in each of these positions.

- Finally, the parameters are optimized such that the parametric FRFs (the FRFs of the linearized parametric robot model) match the estimated nonparametric FRFs.

This identification procedure, first suggested in Öhr et al. (2006), will here be described in more detail. Various aspects of the procedure are also treated in Wernholt and Gunnarsson (2006b), Wernholt and Löfberg (2007), Wernholt and Gunnarsson (2007) and Wernholt and Moberg (2007). Using an FRF-based procedure allows for data compression, unstable systems are handled without problems, it is easy to validate the model such that all important resonances are captured, and model requirements in the frequency domain are also easily handled.

The proposed procedure also has some possible problems. The choice of model structure (parameters) and handling local minima in the optimization are problems here as well. In addition comes some difficulties with biased nonparametric FRF estimates due to closed-loop data and nonlinearities. There are also cases when even a small perturbation around a working point can give large variations due to the nonlinearities, which makes a linear approximation inaccurate, e.g., passing through Coulomb friction, backlash, or different parts of a nonlinear stiffness. This can be partly handled by the choice of excitation (e.g., avoid zero velocity to reduce Coulomb friction). Using multiple positions is good for the parameter accuracy as well as for identifiability issues. It will, however, make it harder to use a linear approximation of certain quantities. Consider, for example, the problem of nonlinear stiffness, where a linear approximation will vary between different positions due to gravity and the amplitude of the excitation. It is then impossible to find a linear stiffness that perfectly matches the resonances for all positions. Still, if the nonlinearity can be parameterized and properly linearized in the different positions, those parameters could possibly be identified as well.

The procedure will now be described, starting with the robot model in Section 3, carrying on by describing the nonparametric FRF estimation and the parameter estimation in Sections 4 and 5, respectively. Experimental results are shown in Section 6, and finally some conclusions are drawn in Section 7.

\section{Robot Model}

The robot model described in this section comes from Moberg and Hanssen (2007). A general serial link industrial robot, as in Figure 1, is then modeled by a kinematic chain of rigid bodies, where each rigid body is connected to the 
preceding body by three torsional spring-damper pairs, giving three degrees-offreedom (DOF) to each rigid body. At most one of these DOFs can be actuated, corresponding to a connection of the two rigid bodies by a motor and a gearbox. In this representation, a robot link (always actuated) can consist of one or more rigid bodies. The model equations, described in Moberg and Hanssen (2007), can be written as a nonlinear gray-box model

$$
\begin{aligned}
& \dot{x}(t)=f(x(t), u(t), \theta), \\
& y(t)=h(x(t), u(t), \theta),
\end{aligned}
$$

with state vector $x(t)$, input vector $u(t)$, output vector $y(t)$, and nonlinear functions $f(\cdot)$ and $h(\cdot)$ that describe the dynamics. The rigid body parameters are assumed to be known and $\theta$ is a vector of unknown parameters for (mainly) springs and dampers. See the previous section for examples of other unknown parameters to include.

\section{FRF Estimation}

As a first step toward the parameter identification, estimates of the nonparametric FRF in a number of positions are needed. These are obtained by performing experiments where the robot is moved into a position and a speed reference signal is fed to the robot controller. The resulting motor torques (actually the torque reference to the torque controller) and angular positions are sampled and stored. The measured angular positions are then filtered and differentiated to obtain estimates of the motor angular speeds, which are here considered as the output signals.

The open-loop system to be identified is unstable, which makes it necessary to collect data while the robot controller is running in closed loop. Consider therefore the setup in Figure 2, where the controller takes as input the difference between the reference signal $r$ and the measured and sampled output $y$, and $u$ is the input. The disturbance, $v$, contains various sources of noise and disturbances. An experimental control system is used, which enables the use of off-line computed reference signals for each motor controller.

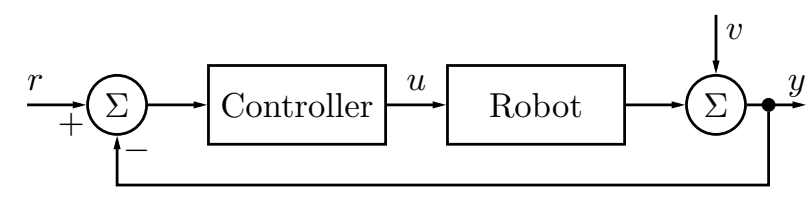

Figure 2: Closed-loop measurement setup.

To avoid leakage effects in the discrete Fourier transform (DFT), which is used by the estimation method, the excitation signal, $r$, is assumed to be periodic, with $N_{P}$ samples in each period, and an integer number of periods of the steady-state response are collected. The nonparametric FRF estimate $\widehat{G}\left(\omega_{k}\right) \in \mathbb{C}^{n \times n}$ (assuming $n$ inputs and outputs) is calculated from a block of $n$ experiments like (Pintelon and Schoukens, 2001, p. 61)

$$
\widehat{G}\left(\omega_{k}\right)=\mathbf{Y}\left(\omega_{k}\right) \mathbf{U}^{-1}\left(\omega_{k}\right),
$$


where the $n$ columns of $\mathbf{Y}\left(\omega_{k}\right)$ and $\mathbf{U}\left(\omega_{k}\right)$ contain the DFT of the sampled data from the $n$ experiments. See also, e.g., Wernholt and Gunnarsson (2007) and Wernholt and Moberg (2007) for other FRF estimators for multivariable systems.

As excitation, an orthogonal random phase multisine signal (Dobrowiecki and Schoukens, 2007) is used, which here gives

$$
\mathbf{R}\left(\omega_{k}\right)=\mathbf{R}_{\text {diag }}\left(\omega_{k}\right) \mathbf{T},
$$

where $\mathbf{R}_{\text {diag }}\left(\omega_{k}\right)$ is a diagonal matrix

$$
\mathbf{R}_{\text {diag }}\left(\omega_{k}\right)=\operatorname{diag}\left\{R_{1}\left(\omega_{k}\right), \ldots, R_{n}\left(\omega_{k}\right)\right\},
$$

and $\mathbf{T}$ is an orthogonal matrix

$$
\mathbf{T}_{i l}=e^{\frac{2 \pi j}{n}(i-1)(l-1)},
$$

with $\mathbf{T T}^{H}=n I$. Each $R_{l}\left(\omega_{k}\right)$ is the DFT of a random phase multisine signal, which in the time domain can be written as

$$
r(t)=\sum_{k=1}^{N_{f}} A_{k} \cos \left(\omega_{k} t+\phi_{k}\right),
$$

with amplitudes $A_{k}$, frequencies $\omega_{k}$ chosen from the grid $\left\{\omega_{k}=\frac{2 \pi k}{N_{P} T_{s}}, k=\right.$ $\left.1, \ldots, \frac{N_{p}}{2}-1\right\}\left(N_{P}\right.$ even) with $T_{s}$ the sampling period, and random phases $\phi_{k}$ uniformly distributed on the interval $[0,2 \pi)$. Using the orthogonal multisine signal in closed loop corresponds to an optimal experiment design given output amplitude constraints.

The selection of frequencies as well as the amplitude spectrum will affect the parameter estimation in the next step. Using too many frequencies will give a low signal-to-noise ratio, which increases both the bias and the variance in the nonparametric FRF estimate. The amplitude spectrum should also reflect the sensitivity for the unknown parameters $\left(\mathrm{cf.} \Psi_{0}^{(i)}(k)\right.$ in $\left.(5)\right)$, at least such that the unknown parameters influence the parametric FRF for the selected frequencies.

The nonparametric FRF estimate can be improved by averaging over multiple blocks and/or periods. The covariance matrix can then also be estimated. For a linear system, averaging over different periods is sufficient, whereas for a nonlinear system, it is essential to average over blocks where $\mathbf{R}_{\text {diag }}$ in each block should have different realizations of the random phases. The reason is that nonlinearities otherwise will distort the estimate and give a too low uncertainty estimate, see Pintelon and Schoukens (2001, Chap. 3) and Schoukens et al. (2005).

For the industrial robot, the nonlinearities cause large distortions and averaging over multiple blocks is therefore important (Wernholt and Gunnarsson, $2006 \mathrm{~b}$ ). For the same reason, one should only excite odd frequencies (only $\omega_{k}=2 \pi(2 k+1) /\left(N_{P} T_{s}\right)$ in $\left.(3)\right)$, see Schoukens et al. (2005).

\section{Parameter Estimation}

When the FRFs have been estimated from data, the next step is to linearize the nonlinear model (1) in the same positions and calculate the parametric FRFs, 
$G^{(i)}\left(\omega_{k}, \theta\right), i=1, \ldots, Q$. A cost function $V(\theta)$ is then formed, measuring the (weighted) discrepancy between the parametric FRF and the estimated nonparametric FRF for all the $Q$ positions. This cost function is finally minimized to identify the unknown parameters.

First, two different parameter estimators will be analyzed and compared. Next, the selection of optimal positions for the experiments is treated, and finally, the solution of the optimization problem is discussed.

Remark 1 Note that the parametric FRF, $G^{(i)}\left(\omega_{k}, \theta\right)$, is a function of the nonlinear gray-box model (1) such that for each parameter vector $\theta$ during the minimization, (1) is linearized in $\left(x_{0}^{(i)}, u_{0}^{(i)}\right)$ before calculating the FRF.

\subsection{Estimators}

\subsubsection{Weighted Nonlinear Least Squares (NLS) Estimator}

The NLS estimator is given by

$$
\begin{gathered}
\hat{\theta}_{N_{f}}^{\mathrm{NLS}}=\arg \min _{\theta \in \Theta} V_{N_{f}}^{\mathrm{NLS}}(\theta), \\
V_{N_{f}}^{\mathrm{NLS}}(\theta)=\sum_{i=1}^{Q} \sum_{k=1}^{N_{f}}\left[\mathcal{E}^{(i)}(k, \theta)\right]^{H}\left[\Lambda^{(i)}(k)\right]^{-1} \mathcal{E}^{(i)}(k, \theta), \\
\mathcal{E}^{(i)}(k, \theta)=\operatorname{vec}\left(\widehat{G}^{(i)}\left(\omega_{k}\right)\right)-\operatorname{vec}\left(G^{(i)}\left(\omega_{k}, \theta\right)\right),
\end{gathered}
$$

with $\Lambda^{(i)}(k)$ a Hermitian $\left(\Lambda=\Lambda^{H}\right)$ weighting matrix, and $(\cdot)^{H}$ denoting complex conjugate transpose. The asymptotic properties $\left(N_{f} \rightarrow \infty\right)$ of this estimator will be derived in the following theorem.

Theorem 1 Consider the NLS estimator (4) and assume that:

1. $\widehat{G}^{(i)}\left(\omega_{k}\right)=G^{(i)}\left(\omega_{k}, \theta_{0}\right)+\eta^{(i)}\left(\omega_{k}\right)$ with $\operatorname{vec}\left(\eta^{(i)}\left(\omega_{k}\right)\right)$ a zero mean circular complex random vector, independent over $i$ and $\omega_{k}$, with covariance matrix $\Lambda_{0}^{(i)}\left(\omega_{k}\right)$.

2. $\Theta$ is a compact set where $V_{N_{f}}^{N L S}(\theta)$ and its first-and second-order derivatives are continuous for any value of $N_{f}$.

3. For $N_{f}$ large enough, the expected value of $V_{N_{f}}^{N L S}(\theta)$ has a unique global minimum in $\Theta$.

The estimator $\hat{\theta}_{N_{f}}^{N L S}$ will then converge to $\theta_{0}$ as $N_{f} \rightarrow \infty$ and $\sqrt{N_{f}}\left(\hat{\theta}_{N_{f}}^{N L S}-\theta_{0}\right)$ is asymptotically Normal distributed with covariance matrix $P_{\theta}$,

$$
\begin{aligned}
P_{\theta}=\frac{1}{2}\left[\frac{1}{N_{f}} \sum_{i=1}^{Q}\right. & \left.\sum_{k=1}^{N_{f}} \Re\left\{\overline{\Psi_{0}^{(i)}(k)} \Xi^{(i)}(k)\left[\Psi_{0}^{(i)}(k)\right]^{T}\right\}\right]^{-1} \\
\times & {\left[\frac{1}{N_{f}} \sum_{i=1}^{Q} \sum_{k=1}^{N_{f}} \Re\left\{\overline{\Psi_{0}^{(i)}(k)} \Sigma^{(i)}(k)\left[\Psi_{0}^{(i)}(k)\right]^{T}\right\}\right] } \\
\times & {\left[\frac{1}{N_{f}} \sum_{i=1}^{Q} \sum_{k=1}^{N_{f}} \Re\left\{\overline{\Psi_{0}^{(i)}(k)} \Xi^{(i)}(k)\left[\Psi_{0}^{(i)}(k)\right]^{T}\right\}\right]^{-1}, }
\end{aligned}
$$


with the Jacobian matrix $\left[\Psi_{0}^{(i)}(k)\right]^{T}=\left.\frac{\partial \operatorname{vec}\left(G^{(i)}\left(\omega_{k}, \theta\right)\right)}{\partial \theta}\right|_{\theta=\theta_{0}}, \overline{(\cdot)}$ denoting complex conjugate, and

$$
\begin{aligned}
& \Xi^{(i)}(k)=\left[\Lambda^{(i)}\left(\omega_{k}\right)\right]^{-1}, \\
& \Sigma^{(i)}(k)=\left[\Lambda^{(i)}\left(\omega_{k}\right)\right]^{-1} \Lambda_{0}^{(i)}\left(\omega_{k}\right)\left[\Lambda^{(i)}\left(\omega_{k}\right)\right]^{-1} .
\end{aligned}
$$

The covariance is minimized by using the optimal weights

$$
\Lambda^{(i)}\left(\omega_{k}\right)=\Lambda_{0}^{(i)}\left(\omega_{k}\right)
$$

which also simplifies (5) to

$$
P_{\theta}=\frac{1}{2}\left[\frac{1}{N_{f}} \sum_{i=1}^{Q} \sum_{k=1}^{N_{f}} \Re\left\{\overline{\Psi_{0}^{(i)}(k)}\left[\Lambda^{(i)}\left(\omega_{k}\right)\right]^{-1}\left[\Psi_{0}^{(i)}(k)\right]^{T}\right\}\right]^{-1} .
$$

Proof 1 Follows from fairly straightforward calculations using Theorem 7.21 in Pintelon and Schoukens (2001).

Note that in addition to the mentioned assumptions, there are some technical details for the asymptotic normality that $\eta^{(i)}\left(\omega_{k}\right)$ has uniformly bounded absolute moments of order $4+\epsilon$ with $\epsilon>0$. See Pintelon and Schoukens (2001, Theorem 7.21) for details.

\subsubsection{Weighted Logarithmic Least Squares (LLS) Estimator}

For systems with a large dynamic range, the NLS estimator may become illconditioned. The weighted logarithmic least squares (LLS) estimator has been suggested as an alternative (Pintelon and Schoukens, 2001, pp. 206-207)

$$
\begin{gathered}
\hat{\theta}_{N_{f}}^{\mathrm{LLS}}=\arg \min _{\theta} V_{N_{f}}^{\mathrm{LLS}}(\theta), \\
V_{N_{f}}^{\mathrm{LLS}}(\theta)=\sum_{i=1}^{Q} \sum_{k=1}^{N_{f}}\left[\mathcal{E}^{(i)}(k, \theta)\right]^{H}\left[\Lambda^{(i)}(k)\right]^{-1} \mathcal{E}^{(i)}(k, \theta), \\
\mathcal{E}^{(i)}(k, \theta)=\log \operatorname{vec}\left(\widehat{G}^{(i)}\left(\omega_{k}\right)\right)-\log \operatorname{vec}\left(G^{(i)}\left(\omega_{k}, \theta\right)\right),
\end{gathered}
$$

where $\log G=\log |G|+j \arg G$. This estimator has improved numerical stability and is particularly robust to outliers in the measurements. However, from a theoretical point of view, the estimator is inconsistent $\left(\lim _{N_{f} \rightarrow \infty} \hat{\theta}_{N_{f}}^{\text {LLS }} \neq \theta_{0}\right)$. The bias can be neglected if the signal-to-noise ratio $\left(\operatorname{vec}(\widehat{G})\right.$ vs. $\left.\sqrt{\operatorname{diag}\left\{\Lambda_{0}\right\}}\right)$ is large enough (at least $10 \mathrm{~dB}$ according to Pintelon and Schoukens, 2001, p. 207).

Similarly to Theorem 1, one can show that the covariance matrix, using the LLS estimator (7), is approximately given by (5) with

$$
\begin{aligned}
& \Xi^{(i)}(k)=\left[G_{d}^{(i)}\left(\omega_{k}, \theta_{0}\right) \Lambda^{(i)}\left(\omega_{k}\right)\left[G_{d}^{(i)}\left(\omega_{k}, \theta_{0}\right)\right]^{H}\right]^{-1}, \\
& \Sigma^{(i)}(k)=\Xi^{(i)}(k) \Lambda_{0}^{(i)}\left(\omega_{k}\right) \Xi^{(i)}(k),
\end{aligned}
$$

and $G_{d}^{(i)}\left(\omega_{k}, \theta_{0}\right)=\operatorname{diag}\left\{\operatorname{vec}\left(G^{(i)}\left(\omega_{k}, \theta_{0}\right)\right)\right\}$. Using the optimal weights

$$
\Lambda^{(i)}\left(\omega_{k}\right)=\left[G_{d}^{(i)}\left(\omega_{k}, \theta_{0}\right)\right]^{-1} \Lambda_{0}^{(i)}\left(\omega_{k}\right)\left[G_{d}^{(i)}\left(\omega_{k}, \theta_{0}\right)\right]^{-H},
$$

gives approximately the same covariance as for the NLS estimator. 


\subsubsection{Selection of Weights}

Even if the covariance is minimized by using the optimal weights, the choice of weights will in general deviate from the optimal ones for a number of reasons. Firstly, the true covariance matrix $\Lambda_{0}^{(i)}\left(\omega_{k}\right)$ is usually not known so the user must instead be content with an estimated covariance matrix $\hat{\Lambda}_{0}^{(i)}\left(\omega_{k}\right)$. Secondly, the weights also reflect where the user requires the best model fit. This is important in case the model is unable to describe every detail in the measurements. The bias-inclination will then be small for frequencies, elements, and positions where the weights $\left[\Lambda^{(i)}\left(\omega_{k}\right)\right]^{-1}$ are large.

For a resonant system, it is often easier to use the LLS estimator in the way that even constant weights will make sure that both resonances and antiresonances are matched by the model. This is due to the fact that the logarithm in the LLS estimator inherently gives the relative error, compared to the absolute error when using the NLS estimator. With the NLS estimator, the antiresonances are easily missed if not choosing large weights at those frequencies.

\subsection{Optimal Positions}

Given a nonlinear gray-box model (1), the information about the unknown parameters will differ between nonparametric FRF estimates in different positions. Therefore, given a limited total measurement time, one should perform experiments in the position(s) that contribute the most to the information about the unknown parameters. In Wernholt and Löfberg (2007), this problem is formulated as follows: Assume a set of $Q_{c}$ candidate positions. Determine the number of experiments to be performed in each position $\left(m_{i}\right.$ experiments in position $i$ ) such that the parameter uncertainty is minimized, given a total of $M=\sum_{i=1}^{Q_{c}} m_{i}$ experiments. Determining the values $m_{i}, i=1, \ldots, Q_{c}$, is a combinatorial experiment design problem which relatively quickly will become intractable when $Q_{c}$ is large. If $M$ is not too small, a good approximate solution can be found by relaxing the constraint that each $m_{i}$ should be an integer. This relaxed problem is convex, which enables the global optimum to be found. In the paper Wernholt and Löfberg (2007), it is also shown that the experiment design is efficiently solved by considering the dual problem. The candidate positions are obtained by griding the workspace. Given thousands of candidate positions, only a few positions typically have a nonzero $m_{i}$ in the optimum. See Wernholt and Löfberg (2007) for details and examples.

\subsection{Solving the Optimization Problem}

The minimization problem to be solved, (4) or (7), is unfortunately non-convex. Here, the problem is solved using fminunc in MatlaB, which is a gradientbased method which only returns a local optimum. Due to the existence of local minima, a good initial parameter vector, $\theta_{\text {init }}$, is important. The problem is solved for a number of random perturbations around $\theta_{\text {init }}$ in order to avoid local minima. Or, alternatively stated, to obtain a local minimum which is good enough for the purpose of the model. The quality of the resulting model, as well as problems with local minima and identifiability properties, depend on the choices of estimator, weights, and position(s) for the experiments. This will be illustrated in the next section. 


\section{Experimental Results}

The described identification procedure from the previous sections will here be used for the identification of an industrial robot from the ABB IRB6600 series using an experimental controller. A nonlinear gray-box model with 26 unknown parameters is used. The nonparametric FRFs are estimated in the 15 optimal positions from Wernholt and Löfberg (2007) by using an odd orthogonal random phase multisine signal with a flat amplitude spectrum as excitation and averaging over a number of blocks. The parameters are then estimated using the following estimators:

LLSM15U: LLS estimator, $Q=15$, only magnitude $(\log |G|)$, user-defined weights.

LLS15U: LLS estimator, $Q=15$, user-defined weights.

LLS150: LLS estimator, $Q=15$, optimal weights.

NLS15U: NLS estimator, $Q=15$, user-defined weights.

NLS150: NLS estimator, $Q=15$, optimal weights.

LLS1U: LLS estimator, $Q=1$, user-defined weights.

For simplicity only diagonal weights $\left[\Lambda^{(i)}\left(\omega_{k}\right)\right]^{-1}$ are considered ${ }^{1}$. The userdefined weights are constant for each element in the FRF, the same for all positions, zero for low frequencies where the nonparametric FRF is uncertain, and lower for the non-diagonal elements in the FRF. The optimal weights are calculated from (6) and (8), using the estimated covariance $\hat{\Lambda}_{0}^{(i)}\left(\omega_{k}\right)$ and $G^{(i)}\left(\omega_{k}, \theta_{0}\right) \approx \widehat{G}^{(i)}\left(\omega_{k}\right)$. The optimal weights often turn out to be small at the resonances and anti-resonances due to a larger relative error in the nonparametric FRF estimate at those frequencies. For the LLS1U estimator, the single position with the smallest theoretical parameter covariance is used.

To assess the sensitivity to the initial parameter vector, $\theta_{\text {init }}, 100$ optimizations are performed for each of the 6 estimators, using randomly perturbed initial parameters, $\theta_{\text {init }}^{[l]}, l=1, \ldots, 100$ (the same for all estimators). Each element in $\theta_{i n i t}^{[l]}$ is obtained by multiplying the corresponding element in $\theta_{\text {init }}$ by $10^{\varphi}$, where $\varphi$ is a random number from a uniform distribution on the interval $[-1,1]$.

To evaluate the resulting 600 models, the same cost function is used for all models. The LLS cost, $V^{\mathrm{LLS}}(\theta)$, is calculated with optimal weights and userdefined weights, which can be seen in Figures 3 and 4 , respectively. The cost varies quite much between the different estimators. What is more important is the trend over the different optimizations. The first three estimators tend to be much more robust to varying initial parameters.

To compare the number of reasonable models, some measure is needed. Since the optimal weights are small at the resonances and anti-resonances, Figure 3 is not so well suited for judging if resonances (and anti-resonances) are accurately modeled or not. Consider therefore Figure 4. When comparing the FRFs of

\footnotetext{
${ }^{1}$ This means that, e.g., (4b) can be rewritten as $V_{N_{f}}^{\mathrm{NLS}}(\theta)=\sum_{i=1}^{Q} \sum_{k=1}^{N_{f}} \sum_{m=1}^{n_{y}} \sum_{n=1}^{n_{u}}\left|\widehat{G}_{m n}^{(i)}\left(\omega_{k}\right)-G_{m n}^{(i)}\left(\omega_{k}, \theta\right)\right|^{2} W_{m+(n-1) n_{y}}^{(i)}\left(\omega_{k}\right)$, where $W^{(i)}\left(\omega_{k}\right)$ is the diagonal of $\left[\Lambda^{(i)}\left(\omega_{k}\right)\right]^{-1}$.
} 


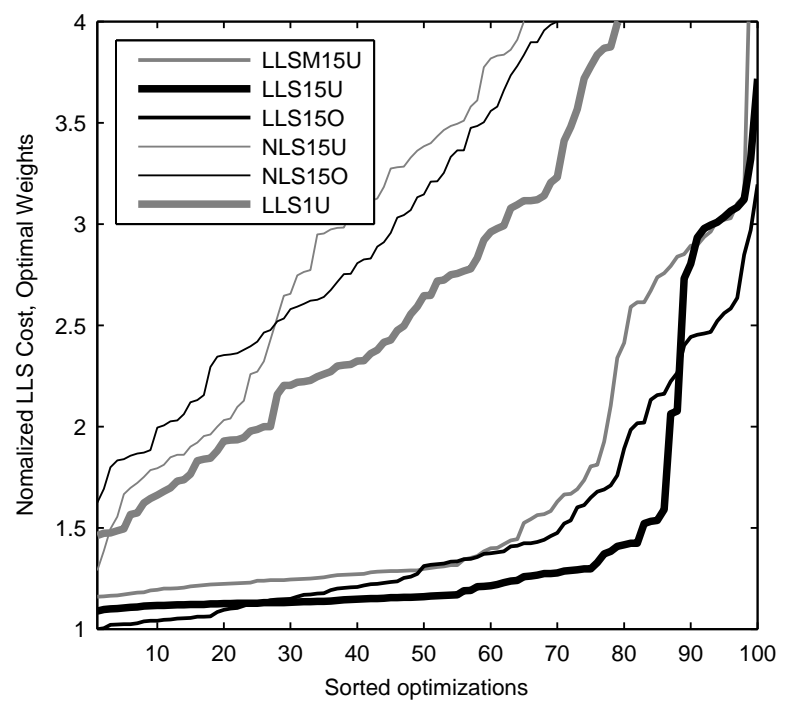

Figure 3: Normalized LLS cost with optimal weights for all initial parameters and all estimators.

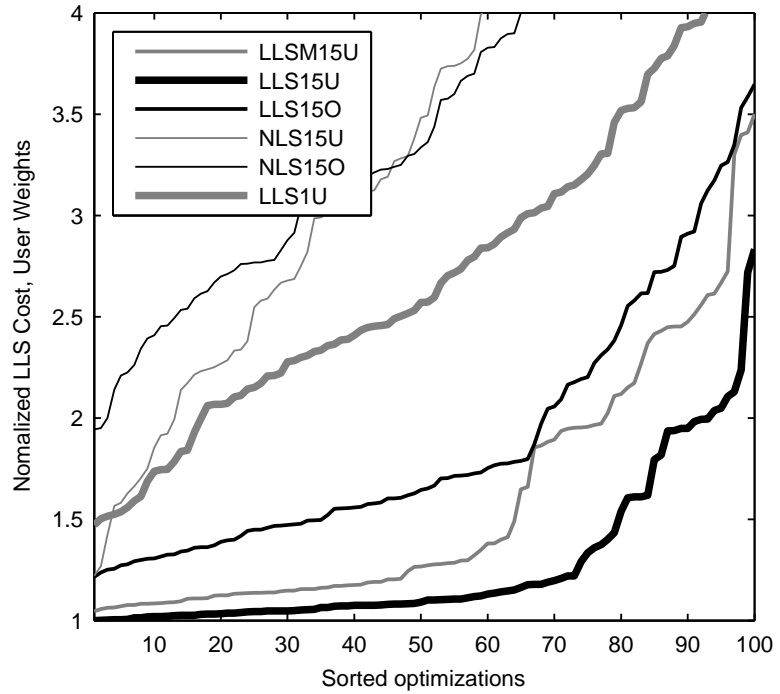

Figure 4: Normalized LLS cost with user-defined weights for all initial parameters and all estimators. 
Table 1: Statistics for the LLS15U estimator, where the first five columns are obtained using the 31 best models in Figure 4, and the last column is calculated for the best model, using (5).

\begin{tabular}{|l|l|l|l|l|l|l|}
\hline$\theta_{i}$ & min & median & max & mean & std & std $_{\text {th }}$ \\
\hline$k_{1}$ & 0.97 & 1.01 & 1.05 & 1.01 & 0.020 & 0.0069 \\
\hline$k_{2}$ & 0.99 & 1.02 & 1.04 & 1.02 & 0.012 & 0.0081 \\
\hline$k_{3}$ & 0.061 & 1.00 & 3.67 & 1.10 & 0.66 & 0.103 \\
\hline$k_{4}$ & 0.72 & 0.99 & 1.26 & 0.97 & 0.13 & 0.042 \\
\hline$c_{1}$ & 0.84 & 0.99 & 1.45 & 1.00 & 0.12 & 0.037 \\
\hline$c_{2}$ & 0.24 & 1.04 & 1.51 & 0.99 & 0.30 & 0.056 \\
\hline$c_{3}$ & 0.16 & 0.55 & 7.05 & 1.57 & 1.93 & 0.865 \\
\hline$c_{4}$ & 0.048 & 0.44 & 34.1 & 2.27 & 6.14 & 0.186 \\
\hline
\end{tabular}

the parametric models with the estimated nonparametric FRFs, the models usually miss important resonances when the normalized cost in Figure 4 exceeds approximately 1.5. That gives the following percentage of reasonable models (out of the 100 models): LLS15U, 80\%, LLSM15U, 64\%, LLS150, 35\%, NLS15U, $3 \%$, LLS1U, $2 \%$, and NLS150, $0 \%$. These numbers are only approximate since the same cost can be achieved if one resonance is missed completely but all others are accurate, and if many resonances are only modeled with moderate accuracy. The latter is often the case for the LLS150 estimator since the exact location of the resonances, as well as their damping, are not so important when using the optimal weights.

To further evaluate the estimated models, the parameter variation is studied. The spring parameters of the gearboxes and the arm structure as a function of the sorted optimizations can be seen in Figures 5 and 6, respectively. These parameters are normalized by the best LLS15U model. One immediately notes that the arm structure springs are harder to estimate, in particular one of them. This parameter does not influence the FRF that much in the selected frequency interval and is therefore hard to estimate. Considering the number of optimizations with estimated parameters inside the interval $[0.5,2]$ (black lines in the figures) gives the following percentage of reasonable models (out of the 100 models): LLS15U, $62 \%$, LLSM15U, $43 \%$, LLS150, $21 \%$, NLS15U, $2 \%$, LLS1U, $2 \%$, and NLS150, $0 \%$. One arm structure parameter is excluded in these numbers, but the LLS15U estimator actually manages to accurately estimate the 12 spring parameters in $12 \%$ of the optimizations. The dampers are unfortunately much harder to accurately estimate, as can be seen in Figure 7. Some of the damping parameters fluctuate quite much even among the best models.

The LLS15U estimator is further analyzed by computing the theoretical parameter uncertainty from (5) for the model with the lowest $V^{\text {LLS }}$ cost, as well as the statistics for the best 31 models, i.e., all models with a cost less than 1.05 in Figure 4. Statistics for 8 representative springs $k_{i}$ and dampers $d_{i}$ are shown in Table 1, where the parameters with both the smallest and the largest uncertainties are included. The conclusions are that the springs are more accurately estimated than the dampers and that the theoretical uncertainty gives a good indication of the quality of the estimated parameters. 

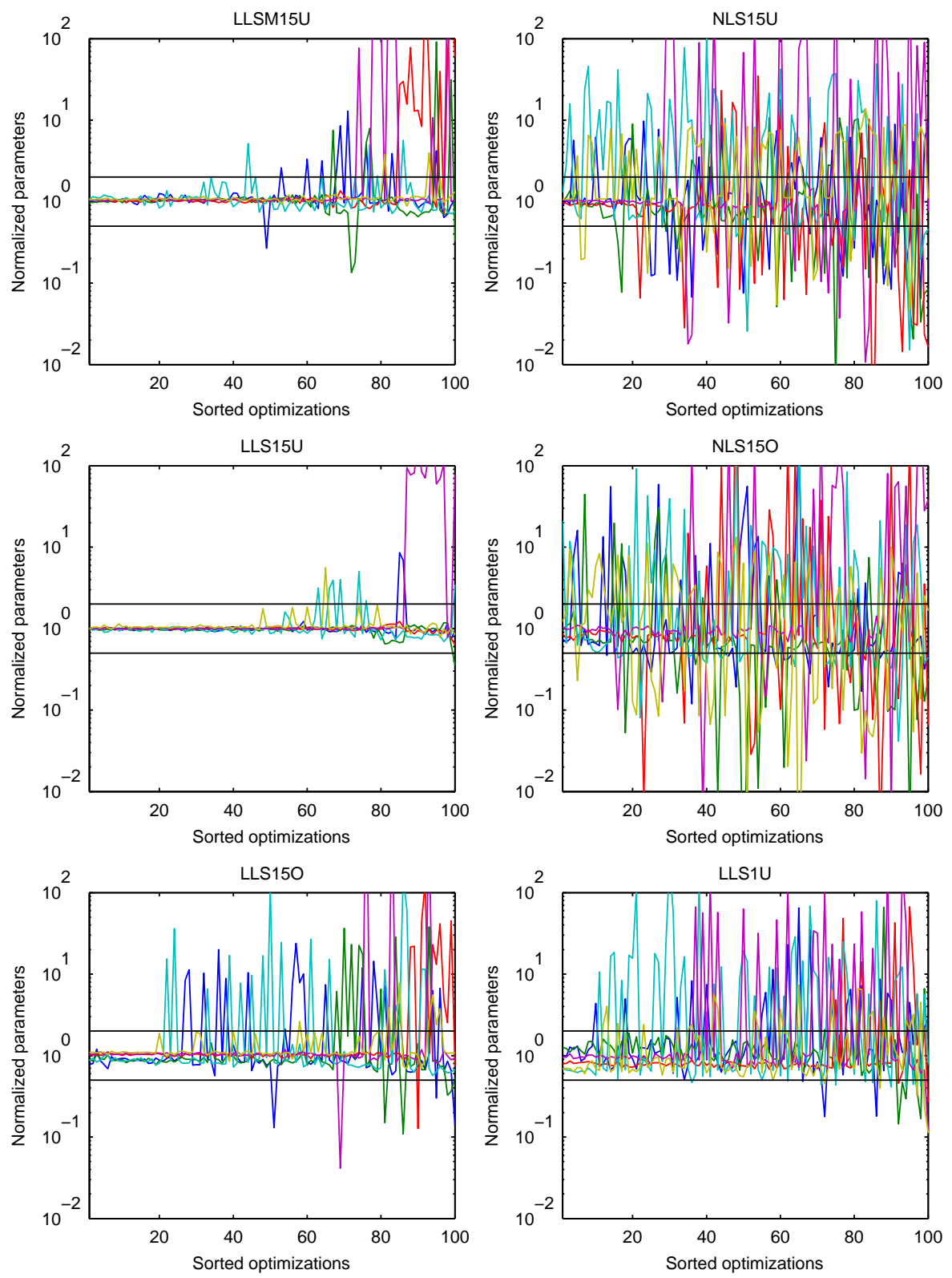

Figure 5: Normalized spring parameters of the gearboxes for the 6 different estimators, sorted according to Figure 4. 

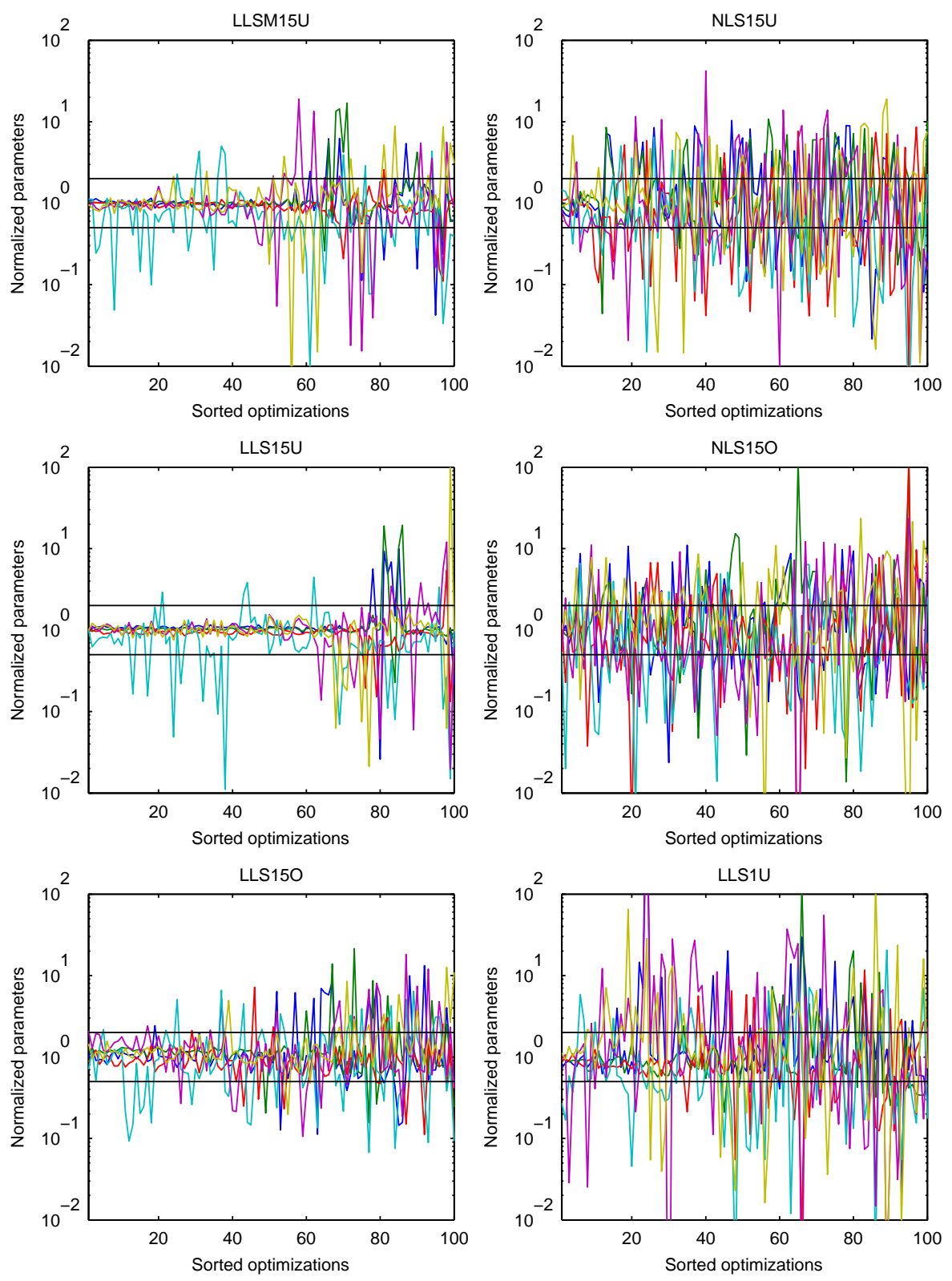

Figure 6: Normalized spring parameters of the arm structure for the 6 different estimators, sorted according to Figure 4. 

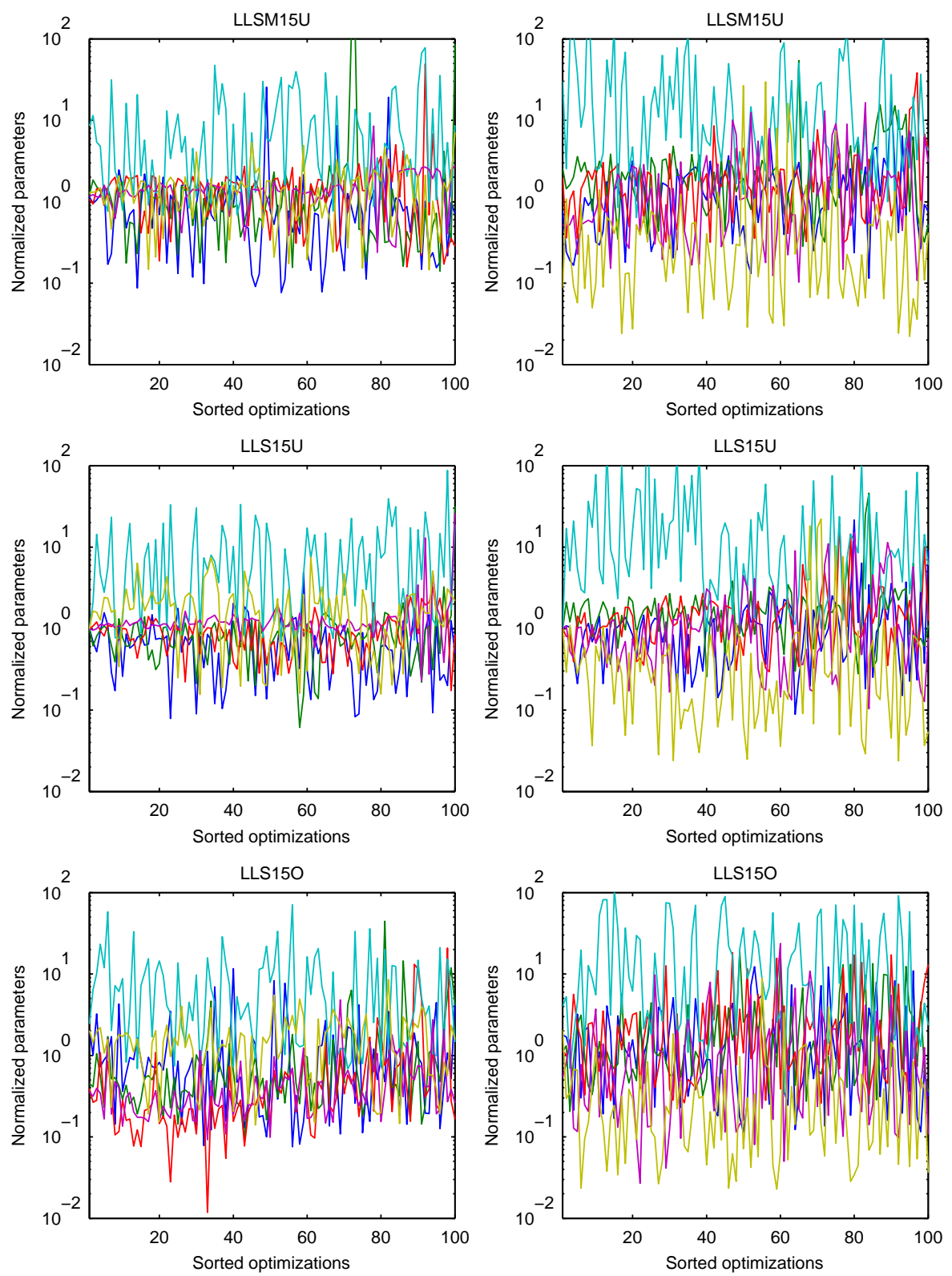

Figure 7: Normalized damping parameters of the gearboxes (left column) and the arm structure (right column) for the estimators LLSM15U, LLS15U and LLS150, sorted according to Figure 4. 


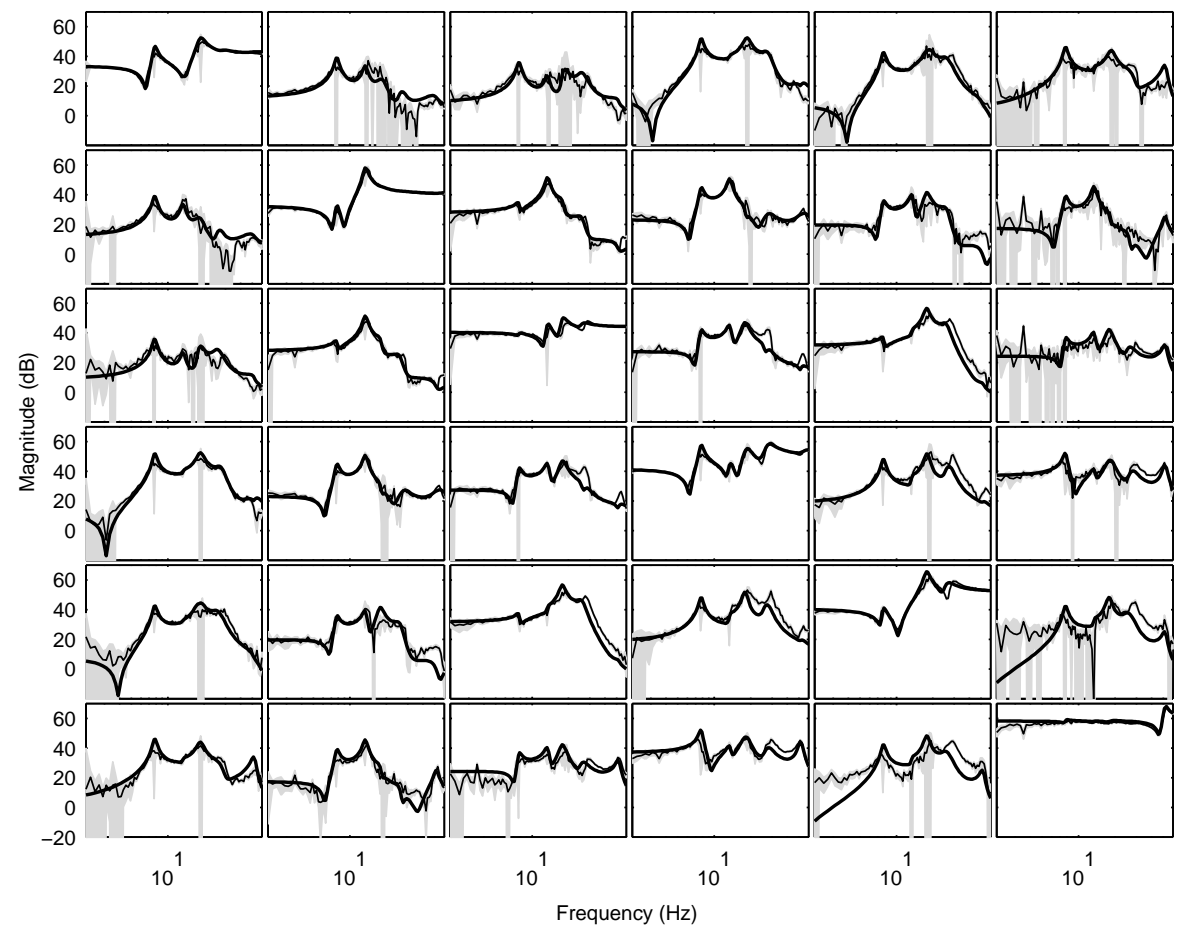

Figure 8: Estimated nonparametric $\operatorname{FRF} \widehat{G}^{(i)}\left(\omega_{k}\right)$ and parametric FRF $G^{(i)}\left(\omega_{k}, \theta\right)$ (the best LLS15U model) in one of the positions. Input: 6 motor torques, output: 6 motor accelerations. Thin line: $\widehat{G}^{(i)}\left(\omega_{k}\right)$, shaded: one standard deviation for $\widehat{G}^{(i)}\left(\omega_{k}\right)$, and thick line: $G^{(i)}\left(\omega_{k}, \theta\right)$.

Figure 8 finally shows the magnitude of the estimated nonparametric FRF and the best parametric model for one of the positions. The identified model gives a good global description of the dynamics in the frequency range of interest.

\section{Concluding Discussion}

This paper has dealt with the problem of estimating unknown elasticity parameters in a nonlinear gray-box model of an industrial robot. An identification procedure has been proposed where the parameters are identified in the frequency domain, using estimates of the nonparametric FRFs for a number of robot configurations/positions. The nonlinear parametric gray-box model is linearized in the same positions and the optimal parameters are obtained by minimizing the discrepancy between the nonparametric and the parametric FRFs. Two different parameter estimators (NLS and LLS) have been analyzed. The estimators, as well as the selection of weights in the estimators, have been evaluated in an experimental study. The conclusions of the experimental study are that:

- the LLS estimator is superior to the NLS estimator for this type of system,

- more than one position is needed in order to get a reasonable estimate, 
- using phase information improves the estimate,

- rough user-defined weights work much better than the theoretically optimal weights,

- gearbox parameters are easier to estimate than arm structure parameters,

- spring parameters are easier to identify than damping parameters, and

- the theoretical uncertainties for the estimated parameters in Table 1 give a good indication of the quality of the estimated parameters.

The uncertainties in the dampers and in some of the springs in the best identified model are quite large, but the resulting global model should anyway be useful for many purposes.

An explanation to the fourth point is that the assumptions in Theorem 1 are violated since the nonparametric FRF estimate has bias errors due to nonlinearities and closed-loop data, and the model is unable to describe every detail of the true system. The weights should, for such a case, primarily be selected to distribute the bias, not to get minimum variance. The theoretically optimal weights are low at the resonance and anti-resonance frequencies (due to uncertainties in the FRF), which in turn give large model errors there.

The fifth point comes as no surprise. The model structure, where all elastic effects in the arm structure are lumped into a few spring-damper pairs, can of course be modified and refined. Both regarding the location of these spring-damper pairs, as well as how many that are needed in order to properly model the system. Identifiability of these added parameters can also be discussed. Maybe additional sensors are needed, e.g., accelerometers attached to the structure, as is the case in experimental modal analysis (Behi and Tesar, 1991; Verboven, 2002).

The main reasons for the large uncertainties in the damping parameters probably are that the system is poorly damped and that the nonparametric FRFs contain errors at the resonances and anti-resonances such that unique damping parameters are hard to find.

A number of areas are still subject to future work. The selection of weights can certainly be improved by combining the user choices and the estimated FRF uncertainty. The selection of frequencies, as well as the amplitude spectrum for the nonparametric FRF estimation can be further improved. An experimental verification of the optimal positions for identification is still interesting to perform. The parameter accuracy and problems with local minima, versus measurement time and excitation energy are also interesting problems to study. Using a frequency-domain method for identification of a nonlinear system has some problems, as was pointed out in Section 2. Therefore, it would be interesting to apply time-domain prediction error methods as a comparison, even though that involves a number of hard problems to tackle, also mentioned in Section 2. A simulation-based study, using a realistic nonlinear model, could also be enlightening.

Finally, to conclude this paper: Identification of industrial robots is a challenging task. Using a general purpose method by pressing a button will almost surely fail. The problem instead requires a combination of tailored identification methods, experiment design, and a skilled user, using all available knowledge about the system. 


\section{References}

A. Albu-Schäffer and G. Hirzinger. Parameter identification and passivity based joint control for a 7DOF torque controlled light weight robot. In Proc. 2001 IEEE International Conference on Robotics and Automation, pages 28522858, Seoul, Korea, May 2001.

A. Albu-Schäffer and G. Hirzinger. State feedback controller for flexible joint robots: A globally stable approach implemented on DLR's light-weight robots. In Proc. 2000 IEEE/RSJ International Conference on Intelligent Robots and Systems, pages 1087-1093, Takamatsu, Japan, October 2000.

C. H. An, C. G. Atkeson, and J. M. Hollerbach. Model-Based Control of a Robot Manipulator. MIT Press, Cambridge, Massachusetts, 1988.

F. Behi and D. Tesar. Parametric identification for industrial manipulators using experimental modal analysis. IEEE Transactions on Robotics and Automation, 7(5):642-652, October 1991.

T. Dobrowiecki and J. Schoukens. Measuring a linear approximation to weakly nonlinear MIMO systems. Automatica, 43(10):1737-1751, October 2007.

R. Johansson, A. Robertsson, K. Nilsson, and M. Verhaegen. State-space system identification of robot manipulator dynamics. Mechatronics, 10(3):403-418, April 2000.

W. Khalil and M. Gautier. Modeling of mechanical systems with lumped elasticity. In Proc. 2000 IEEE International Conference on Robotics and Automation, pages 3964-3969, San Francisco, California, April 2000.

K. Kozlowski. Modelling and identification in robotics. Advances in Industrial Control. Springer, London, 1998.

L. Ljung. System Identification: Theory for the User. Prentice Hall, Upper Saddle River, New Jersey, USA, 2nd edition, 1999.

S. Moberg and S. Hanssen. A DAE approach to feedforward control of flexible manipulators. In Proc. 2007 IEEE International Conference on Robotics and Automation, pages 3439-3444, Roma, Italy, April 2007.

J. Öhr, S. Moberg, E. Wernholt, S. Hanssen, J. Pettersson, S. Persson, and S. Sander-Tavallaey. Identification of flexibility parameters of 6-axis industrial manipulator models. In Proc. ISMA2006 International Conference on Noise and Vibration Engineering, pages 3305-3314, Leuven, Belgium, September 2006 .

R. Pintelon and J. Schoukens. System identification: a frequency domain approach. IEEE Press, New York, 2001.

J. Schoukens, R. Pintelon, T. Dobrowiecki, and Y. Rolain. Identification of linear systems with nonlinear distortions. Automatica, 41(3):491-504, March 2005 .

M. W. Spong. Modeling and control of elastic joint robots. Journal of Dynamic Systems Measurement and Control, 109(4):310-319, December 1987. 
P. Verboven. Frequency-domain system identification for modal analysis. PhD thesis, Vrije Universiteit Brussel, Belgium, May 2002.

E. Wernholt and S. Gunnarsson. Nonlinear identification of a physically parameterized robot model. In Proc. 14th IFAC Symposium on System Identification, pages 143-148, Newcastle, Australia, March 2006a.

E. Wernholt and S. Gunnarsson. Detection and estimation of nonlinear distortions in industrial robots. In Proc. 23rd IEEE Instumentation and Measurement Technology Conference, pages 1913-1918, Sorrento, Italy, April 2006b.

E. Wernholt and S. Gunnarsson. Analysis of methods for multivariable frequency response function estimation in closed loop. In 46th IEEE Conference on Decision and Control, New Orleans, Louisiana, December 2007. Accepted for publication.

E. Wernholt and J. Löfberg. Experiment design for identification of nonlinear gray-box models with application to industrial robots. In 46th IEEE Conference on Decision and Control, New Orleans, Louisiana, December 2007. Accepted for publication.

E. Wernholt and S. Moberg. Experimental comparison of methods for multivariable frequency response function estimation. Technical Report LiTH-ISYR-2827, Department of Electrical Engineering, Linköping University, SE-581 83 Linköping, Sweden, October 2007. Submitted to the 17th IFAC World Congress, Seoul, Korea. 


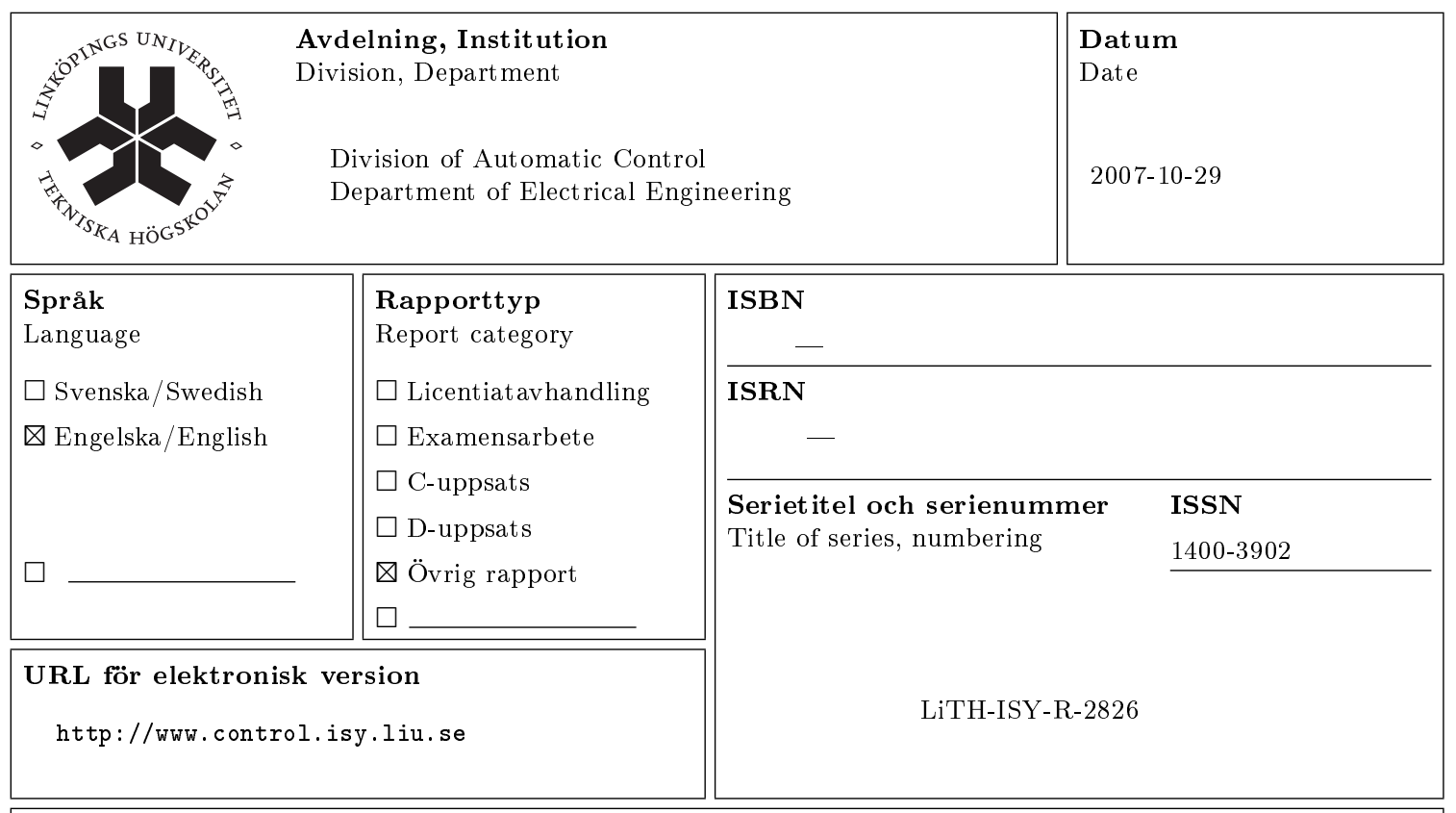

Titel Frequency-Domain Gray-Box Identification of Industrial Robots

Title

(Tite

Författare Erik Wernholt, Stig Moberg

Author

\section{Sammanfattning}

Abstract

This paper considers identification of unknown parameters in elastic dynamic models of industrial robots. Identifying such models is a challenging task since an industrial robot is a multivariable, nonlinear, resonant, and unstable system. Unknown parameters (mainly spring-damper pairs) in a physically parameterized nonlinear dynamic model are identified in the frequency domain, using estimates of the nonparametric frequency response function (FRF) in different robot configurations/positions. The nonlinear parametric robot model is linearized in the same positions and the optimal parameters are obtained by minimizing the discrepancy between the nonparametric FRFs and the parametric FRFs (the FRFs of the linearized parametric robot model). In order to accurately estimate the nonparametric FRFs, the experiments must be carefully designed. The selection of optimal robot configurations for the experiments is also part of the design. Different parameter estimators are compared and experimental results show the usefulness of the proposed identification procedure. The weighted logarithmic least squares estimator achieves the best result and the identified model gives a good global description of the dynamics in the frequency range of interest. 